Check for updates

Cite this: RSC Adv., 2017, 7, 23046

\title{
Multiplicity of shape selection in functionally graded liquid crystalline polymers $\dagger$
}

\author{
Da-Wei Lee, Jayanta Phadikar and M. Ravi Shankar (D)*
}

The synergy of through-thickness gradation in the orientation of the molecular director and the extent of polymerization is shown to offer a framework for controlling shape selection in integral polymer films. Native curvatures are realized under ambient conditions in splayed liquid crystalline polymers that are photopolymerized on anchoring surfaces, while being exposed to the atmosphere. Residual multiaxial polymerization strains drive the spontaneous assembly of a range of geometries (tape springs, helical coils and arches) in strips that are excised from the as-prepared films. Gradients in the director orientation and the cross linking through the thickness enable a temperature dependent structural evolution. Following a moderate temperature rise $\left(<70^{\circ} \mathrm{C}\right)$, the samples are found to intensify their native shapes. However, further temperature rise leads to a relaxation of the strains followed by an eversion of the geometry $\left(>100{ }^{\circ} \mathrm{C}\right)$, which generates curvature orthogonal to that in the native state. This multiplicity in shape selection, which spontaneously emerges without requiring any mechanical training offers a useful framework for actuation and morphing. In a prototypical demonstration, a suitably excised sample is shown to spontaneously jump when placed on a hot-plate as a result of the eversion. Also, when confined in ring-like geometries, hinge-like structures are generated due to the interplay of the imposed bending strains with that existing in the native state. The evolution of the curvatures as a function of temperature offers control over the active hinge/fold and expands the multiplicity of shapes that can be realized.

Received 25th March 2017 Accepted 18th April 2017

DOI: $10.1039 / c 7 r a 03465 b$

rsc.li/rsc-advances accomplished by ultrafine voxels, ${ }^{4}$ incorporation of topological defects $^{5}$ and via hierarchical (through the thickness) variation of the director in monolithic samples. ${ }^{6}$ The through-thickness rotation of the nematic director in twisted-nematic configurations have been exploited to generate thermal transformations of straight, flat strips into an array of helical geometries that are programmable by controlling the offset angle of the director to the long-axis of the strips. ${ }^{6}$ Mediated by the energy balance between stretching and bending in the material, a transition between helical and helicoidal shapes can occur as a function of the width of the sample. ${ }^{7}$ Analogously, in splayed configurations Fig. 1(a), thermomechanical response generates increasing curvatures with increasing temperature. ${ }^{8}$ The thermal responsiveness leading to the generation of such structures can also be achieved using light in azobenzene-functionalized LCP, by exploiting the hierarchical variation of the strains that are generated due to the rotation of the nematic director through the thickness. ${ }^{9}$

Prior research has focused on examining ${ }^{4-6,10-12}$ the evolution of the geometry in LCP strips, typically from a prior flat state following heating or in response to irradiation with light. However, geometrically biasing the prior shape can expand the range of actuation modes. For example, utilizing helical shapes instead of flat strips as the starting point helps achieve large actuation distances and improved work potential in twisted
Department of Industrial Engineering, Swanson School of Engineering, University of Pittsburgh, 3700 O'Hara Street, Pittsburgh, PA 15261, USA. E-mail: ravishm@pitt.edu $\dagger$ Electronic supplementary information (ESI) available. See DOI: $10.1039 / \mathrm{c} 7 \mathrm{ra03465b}$ 
nematic LCP. ${ }^{13}$ Sawa et $a .^{7}$ and Wie et $a .^{10}$ have utilized the anisotropic thermal contraction from the elevated polymerization temperature to ambient conditions to generate chiral (spiral/helicoidal) geometries. The orientation of the nematic director in twisted nematic samples was used to control the geometries. When the chiral shape was heated, it was shown to become flat at an elevated temperature and then was found to invert their chirality with increased heating. Mol et al. ${ }^{8}$ examined the thermomechanical response of splayed LCP, which is characterized by a temperature dependent evaluation of the curvature. The evolution of the geometry was monotonic, where the curvature increased with increasing temperature.

Here, we examine a splayed LCP, which is fabricated with a gradation in the polymerization through the thickness in addition to a rotation of the nematic director. One surface is oriented using an anchoring layer, while the other surface is exposed to atmosphere during the curing. The resulting broken symmetry results in a sample with a native curvature (NC) under ambient conditions, which is a function of the orientation of the nematic director with respect to the long axis of the strip. In addition to presenting counterintuitive thermomechanical responses, the evolution of the geometry offers multimorphism, which offers opportunities for achieving ultrafast actuation and designing morphable structures.

\section{Experimental methods}

Fig. 1(a) illustrates the fabrication approach where the ambient atmosphere is used to simultaneously achieve homeotropic alignment and a gradient in the cross-link density. A splayed orientation is created by photo-polymerizing liquid crystal monomer film coated on planar alignment layer on glass substrate. On the topside liquid crystal monomers are exposed to the oxygen in atmosphere and as a result the polymerization

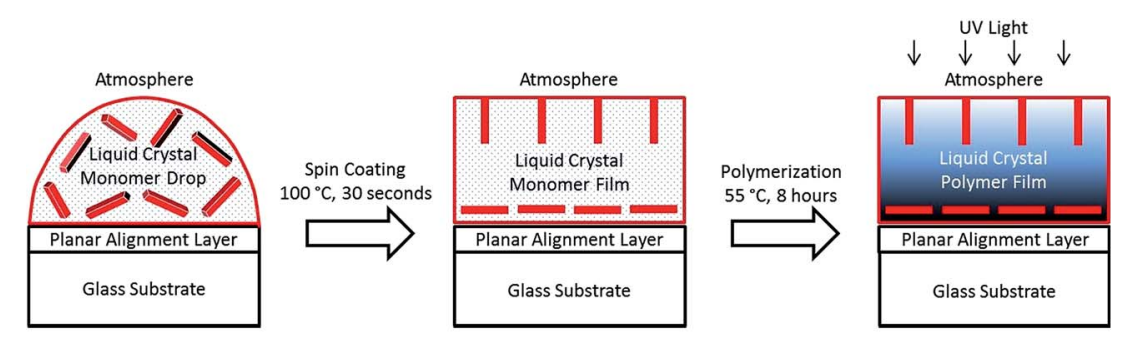

(a)

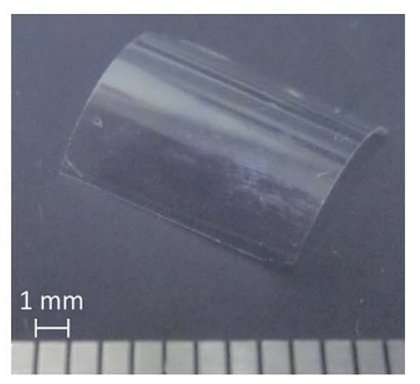

(b)

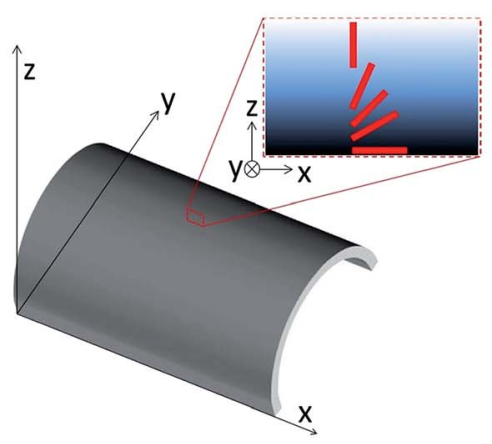

(c)

Fig. 1 (a) Fabrication of functionally-graded liquid crystalline polymers that are characterized by a native curvature under ambient conditions. (b) Curved shape of a $1 \mathrm{~cm} \times 1 \mathrm{~cm} \times 15 \mu \mathrm{m}$ sample after being released from the substrate following polymerization. (c) Illustration of the orientation of the director through the thickness of the sample and its correspondence to the curvature. 
is retarded by the diffusion of oxygen, which depletes free radicals. ${ }^{14}$ As a result, the sample undergoes polymerization in a graded manner, where the underside in contact with the glass polymerizes sooner than that exposed to the air.

To create the samples, the glass substrates were cleaned using a plasma cleaner PDC-32G (Harrick Plasma) for 20 minutes. The planar alignment material composed of $0.5 \mathrm{wt} \%$ solution of nylon Elvamide 8023R (Dupont) in methanol was filtered through $0.2 \mu \mathrm{m}$ PTFE membrane syringe filter (Pall), and spin coated on the glass substrate by spinning at $2000 \mathrm{rpm}$ for 30 seconds. Thus, the substrate was rubbed 30 times by using a velvet cloth. Liquid crystal monomer mixture of acrylatefunctionalized reactive mesogens RMM34C (Merck, proprietary composition characterized by RM257 being the predominant component) was heated to $100{ }^{\circ} \mathrm{C}$, and a droplet was placed on the surface of $8023 \mathrm{R}$ on glass substrate. Spin coating was performed at $1000 \mathrm{rpm}$ for 30 seconds to create monomer film to be $15 \mu \mathrm{m}$. The sample was cooled to a temperature of $55^{\circ} \mathrm{C}$, while remaining exposed to the atmosphere. The planar aligned nematic liquid crystal molecules on the rubbed $8023 \mathrm{R}$ were confirmed using crossed polarizers. $365 \mathrm{~nm}$ UV source generating an intensity of $200 \mathrm{~mW} \mathrm{~cm} \mathrm{~cm}^{-2}$ was used to polymerized the sample for 8 hours, while continuously to be exposed to the atmosphere at $55{ }^{\circ} \mathrm{C}$. The samples were also characterized using polarized optical microscopy.

Attenuated Total Reflection (ATR) Fourier Transform Infrared (FTIR) Spectrometer (Bruker Vertex 70) was used to characterize the film. The transmittance on both sides (homeotropic and planar) of native bending LCP film are measured to characterize the double bond $(\mathrm{C}=\mathrm{C})$ conversion on the surface. The spectrum was measured on a Vertex 70 spectrometer via 64 scans at resolution $4 \mathrm{~cm}^{-1}$ in the range from $600 \mathrm{~cm}^{-1}$ to 4000 $\mathrm{cm}^{-1}$. The PIKE MIRacle ATR accessory was used to conduct the measurement. The planar and the homeotropically aligned sides of sample were placed on the crystal plate along with a pressure clamp to ensure contact, following which the OPUS software was used to collect, subtract the background signal and analyze the data.

\section{Results and discussion}

\subsection{Geometries under ambient conditions}

After curing a square film $1 \mathrm{~cm} \times 1 \mathrm{~cm} \times 15 \mu \mathrm{m}$ was excised from the glass substrate and was found to possess a native curvature at room temperature, as shown in Fig. 1(b). The convex side on top is homeotropically aligned, and the concave side at bottom is the planar one (Fig. 1(a) and (c)). The native curvature is orthogonal to the director on the planar aligned side. This curvature under ambient conditions results from the anisotropic shrinkage in the LCP following the polymerization at the elevated temperature. ${ }^{7,10}$ Residual strains result in the geometry illustrated in Fig. 1(b), when the sample is released from the glass substrate. When the sample is cooled to room temperature the order parameter increases, which leads to (a) on the planar side - tensile strains along the nematic director and compressive strains perpendicular to the nematic director and (b) on the homeotropic side - biaxial compressive strains.
Unlike the homeotropic side, the planar oriented side is constrained by the substrate, which prevents the accommodation of the strains during cooling of the sample. As a result of the broken symmetry in the boundary conditions imposed on the sample, elastic residual stresses are generated on the planar oriented side. The residual strains are correspondingly tensile along the nematic director and compressive perpendicular to it. In contrast, material elements near the homeotropic side exposed to the free surface does not develop residual strains because accommodation of shrinkage strains can occur without constraint. Ideally, the samples under ambient conditions should possess a shape characterized by a finite Gaussian curvature, although it is not entirely apparent in Fig. 1(b). Nonetheless, when strips are excised from this sample as illustrated in Fig. 2, the minimization of the elastic energy results in a shape diversity that is reminiscent of that observed in samples extracted from "saddle-shaped" prestrained polymers. ${ }^{15}$ This shape evolution as a function of the offset angle of the strips in Fig. 2 is spontaneous and points to the residual elastic strains in the materials, which drives shape selection.

Fig. 2(a) illustrates the orientations in which strips were excised from the sample. We observe a one-to-one correspondence between the orientation of the nematic director on the planar side and the morphology of the strips. The $0^{\circ}$ sample corresponds to the director on the planar aligned (patterned) side being parallel to the long-axis of the strip and the $90^{\circ}$ sample corresponds to the director being parallel to the shortaxis. In all cases the other surface is characterized by a homeotropic orientation. The $0^{\circ}$ sample is characterized by a curvature that develops transverse to the nematic director with the homeotropic side corresponding to the surface in tension (Fig. 2(b)). The radius of curvature is measured to be $1.2 \mathrm{~mm}$. The $90^{\circ}$ sample is characterized by the curvature again being transverse to the nematic director with a radius of curvature being $4.2 \mathrm{~mm}$. When a cut is made at an intermediate angle of $50^{\circ}$, a left-handed helix results with a pitch of $11.7 \mathrm{~mm}$ and a diameter of $4.6 \mathrm{~mm}$. The chirality and the geometry of the samples were characterized using multiple samples that were independently created. Fig. 2(c) is used to illustrate the geometry in an easily perceivable manner using paper models with the red illustrating the homeotropic side and green illustrating the planar side.

In order to confirm that the nematic director in the excised samples is indeed varying from homeotropic (the atmosphere side) to planar (the rubbed alignment layer side), polarized-light optical microscopy (POM) and scanning electron microscope (SEM) are used. Under crossed polarizers, the film appears alternatively dark and bright (Fig. 3(a)) when the planar alignment orientation is $0^{\circ}$ and $45^{\circ}$ with respect to the polarizer. The SEM (FEI/Philips XL-30) operated at $2 \mathrm{kV}$ is used to observe the profile through thickness of the film. The methodology outlined in ref. 12 and 16 was used to prepare the sample. A splay distribution is observed from the planar side to the homeotropic side. For illustrative purposes, as a guide for the eye, dashed lines are corresponding to the director as shown in Fig. 3(b). The splay distribution is enabled by the small pre-tilt angle of liquid crystal molecules on the rubbed planar alignment layer. ${ }^{17}$ 

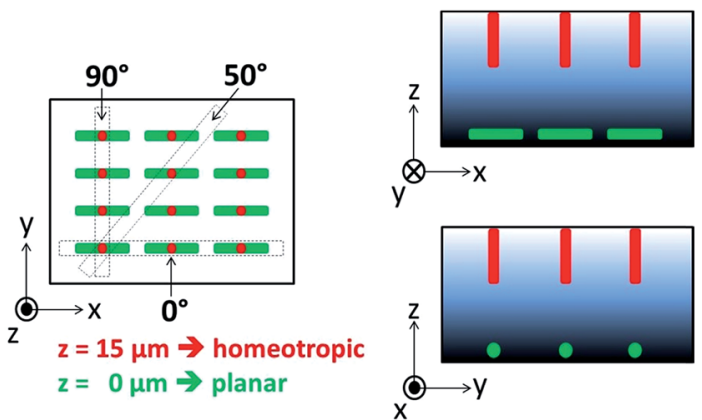

(a)

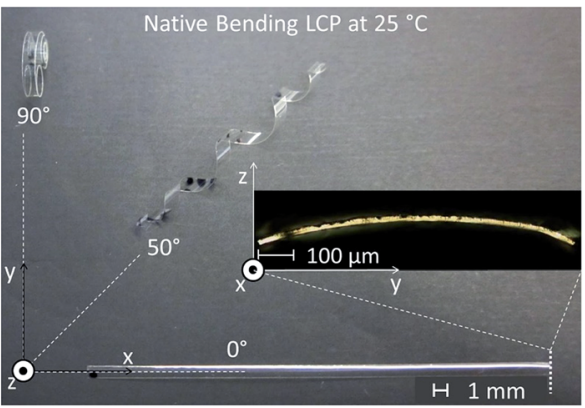

(b)

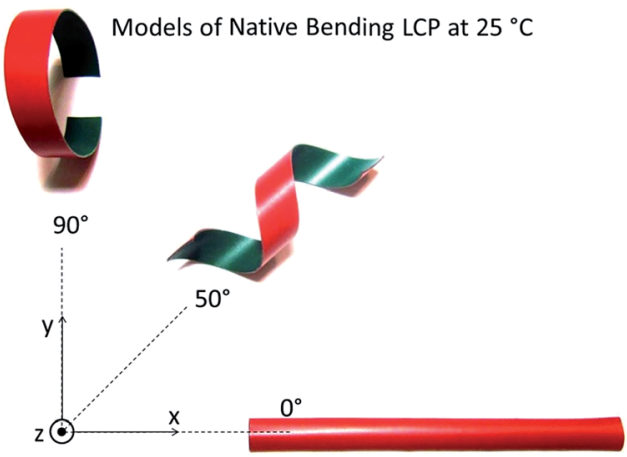

(c)

Fig. 2 (a) Director orientations of the sample and its correspondence to the strips that are excised is illustrated. The planar alignment is achieved at $z=0 \mu \mathrm{m}$, which is hitherto in contact with a rubbed substrate. Homeotropic alignment is on the top surface, where $z=15$ $\mu \mathrm{m}$. The director orientation of planar aligned liquid crystal is defined as $0^{\circ}$, and the cutting angle is increased counterclockwise. (b) At room temperature $25^{\circ} \mathrm{C}$, samples were excised at offset angles of $0^{\circ}, 50^{\circ}$, and $90^{\circ}$ with dimensions of $1 \mathrm{~mm} \times 35 \mathrm{~mm} \times 15 \mu \mathrm{m}$. The resulting strips spontaneously adopt a range of geometries corresponding to a tape spring, a left-handed spiral ribbon, and a circular ribbon respectively. (c) To illustrate the correspondence of the shapes to the homeotropic and planar oriented sides, paper models were created. The red surface represents the homeotropic side, and the green surface represents the planar side.

\subsection{Thermomechanical response}

$1 \mathrm{~mm} \times 35 \mathrm{~mm} \times 15 \mu \mathrm{m}$ strips were excised at various angles as illustrated in Fig. 2(b) and subjected to heat treatment in silicone oil to trace the evolution of their geometry as a function of

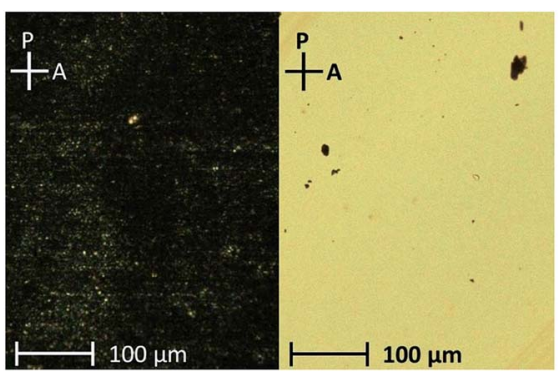

(a)

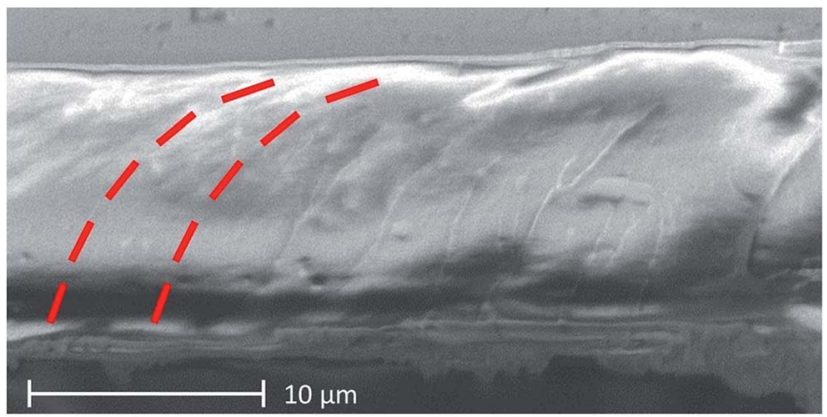

(b)

Fig. 3 (a) Polarized-light optical microscopy (POM) pictures of the NB LCP film in which the planar alignment orientation is $0^{\circ}$ and $45^{\circ}$ with respect to the polarizer. (b) The splay liquid crystal director distribution through the thickness of the film was revealed using the SEM. Red dash lines represent the director, which is intended to be a guide for the eye.

the temperature. Imaging was performed in situ using a camera that continuously recorded the evolution of the geometry. The heating rate was $2{ }^{\circ} \mathrm{C}$ per minute in all experiments.

The thermomechanical responses as a function of the temperature in Fig. 4 illustrate an inherent multimorphism. The following overarching observations emerge:

(a) Heat treatment from ambient to a temperature of $\sim 70{ }^{\circ} \mathrm{C}$ leads to an increase in the magnitude of the strain state characterizing the native curvature at ambient temperature - the tape spring geometry becomes more pronounced $\left(0^{\circ}\right.$ sample: Fig. 4(a)(i-ii)), the helix becomes more tightly coiled $\left(50^{\circ}\right.$ sample: Fig. 4(b)(i-iii)), and the curved arch becomes progressively steeper $\left(90^{\circ}\right.$ sample: Fig. $4(\mathrm{c})$ (i-iii)). The development of this increased curvature is such that the homeotropic side develops greater tensile strains, while the planar oriented side accumulates greater compressive strains. The principal compressive axis on the planar side however, is transverse to the nematic director. This aspect is counter to that observed in splayed liquid crystal polymers, where the principal compressive strains develop along the nematic director. ${ }^{8}$ In ref. 8 , it has been shown that the planar oriented side generates compressive strains along the nematic director, with the principal compressive strain direction coincident with the director. The homeotropic side generates tensile strains and the sample is found to demonstrate a curvature that monotonically increases with increasing temperature. In Fig. 4(b), the helix is left- 


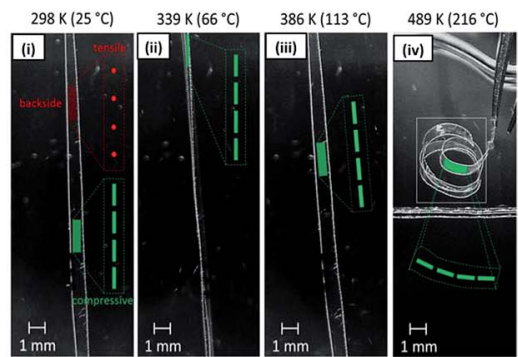

a)
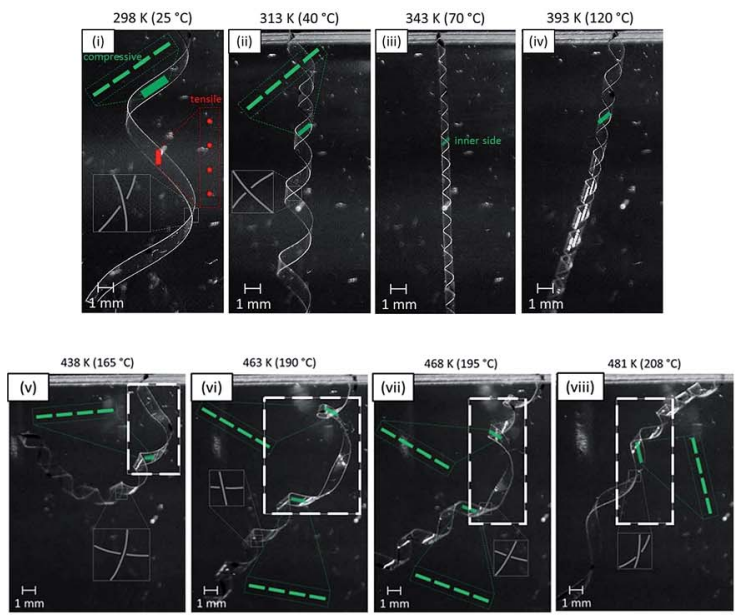

b)

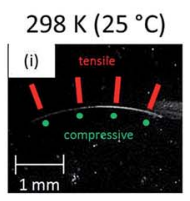

$423 \mathrm{~K}\left(150^{\circ} \mathrm{C}\right)$

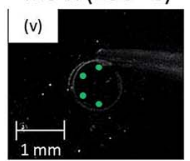

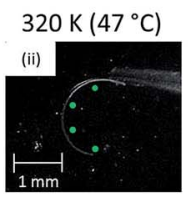

$493 \mathrm{~K}\left(220^{\circ} \mathrm{C}\right)$

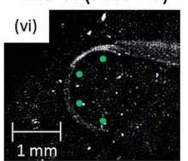

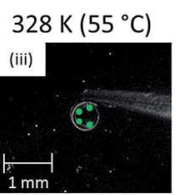

$528 \mathrm{~K}\left(255^{\circ} \mathrm{C}\right)$

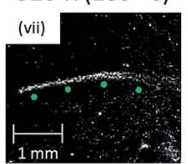

c)

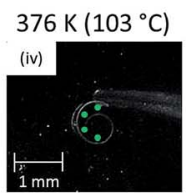

$532 \mathrm{~K}\left(259^{\circ} \mathrm{C}\right)$

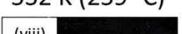

Fig. 4 Thermomechanical response of samples characterized by native curvature is illustrated as a function of the temperature and the offset angle. The red markers illustrate the orientation of the nematic director on the homeotropic side, while the green markers illustrate the orientation of the planar alignment. The markers serve to illustrate the director orientation as a function of the geometry of the sample. Inset boxes in figures ( $a$ and $b$ ) illustrate the crossover of the edges of the strip (in gray), which help visualize the chirality of the twist. (a) $0^{\circ}$ sample is initially in a tape-spring geometry, which first becomes more pronounced and subsequently undergoes eversion. (b) Helical geometry of the $50^{\circ}$ sample that initially coils and then undergoes eversion. Note the box illustrated with a dashed white line, where the eversion of the helix emerges and progresses through the sample. (c) The native curvature of the $90^{\circ}$ sample is shown, which straightens with increasing temperature. However, this sample is not found to undergo eversion.

handed at ambient temperatures and retains this chirality as the temperature is increased (i-iii). The insets drawn in gray in the Fig. 4(b) illustrates the crossover between the edges of the strip. This can help visualize, from the overcrossing and undercrossing of the edges, the chirality (twist) of the helix. Fig. 4(c) illustrates samples excised in the $90^{\circ}$ orientation, where the nematic orientation is homeotropic on one side and perpendicular to the long-axis on the other side. Consistent with the behavior summarized earlier, the homeotropic side is in tension under ambient conditions, while the planar oriented side is in compression. However, the principal direction of the compressive strain is normal to the nematic director. Increasing temperatures Fig. 4(c)(i-iii) show an increase in the magnitude of this strain state, where the sample becomes more tightly coiled.

(b) Continued heat treatment illustrates a reversal of the strain state, which is particularly pronounced $>100{ }^{\circ} \mathrm{C}$. In the case of the tape-spring configuration in Fig. 4(a), the sample is found to become nearly flat as illustrated in Fig. 4(a)(iii). However, further increase in temperature results in the development of curvature orthogonal to the prior native state - the sample undergoes eversion. In Fig. 4(a)(iv), the $0^{\circ}$ sample was found to curl along the long axis, with no observable transverse curvature that hitherto characterized the native state. The homeotropic side is now exclusively in compression, while the planar side is in tension, with the principal direction of the tensile strain coincident with the nematic director. A similar trend characterizes the $50^{\circ}$ sample and $90^{\circ}$ sample. In the $50^{\circ}$ sample (Fig. 4(b)(iii-iv)) increasing temperature is found to uncoil the helix when the temperature increases from 70 to $120{ }^{\circ} \mathrm{C}$. Further temperature increase is found to create a segment where the sample becomes flat. This region is highlighted by a dashed white box in Fig. 4(b)(v-viii). Consistent with the $0^{\circ}$ sample, we find an eversion of the $50^{\circ}$ sample without any change in the chirality of the helix, which remains left-handed. The spiral turns inside-out with the planar oriented side adopting tensile strains while the homeotropic develops compressive strains. The $90^{\circ}$ sample illustrates a comparable trajectory of shape change where Fig. 4(c)(iv) illustrates a reduction in the curvatures, followed by a flattening of the sample with increasing temperatures Fig. 4(c)(iv-viii). At the highest temperatures, the sample is expected develop a transverse curvature with tensile strains along the nematic director. However, we do not explicitly observe the development of this curvature in the images.

This behavior is related to the gradient in the nematic director orientation and its interplay with crosslink density. The material elements near homeotropic side, which are crosslinked considerably less than the planar aligned side, will generate greater magnitudes of strain when subjected to an elevated temperature. In response to moderate heat treatment ( less than $70{ }^{\circ} \mathrm{C}$ ) the greater mobility of the molecular segments with less crosslink density allows greater responsiveness. The strain generation near the homeotropic surface will be one of biaxial tension concomitant with the decrease in the order parameter with increasing temperatures. In contrast, the highly crosslinked material elements in the vicinity of the planar aligned surface will generate much smaller levels of strain, due to the lower mobility of the highly crosslinked polymer chains. Being isolated from the deleterious effects of 
oxygen during the polymerization, the planar aligned underside achieves greater crosslink densities and in doing so becomes less responsive in comparison to the homeotropic side. Thus, the biaxial tension developed in the vicinity of the homeotropic side dominates the thermomechanical response (Fig. 5). The material elements near the planar oriented side remain predominantly unresponsive during this moderate heat treatment. The strain generation under these conditions is incapable of generating any biaxial stretch in the slender films considered here due to the large energy penalty it entails vis$\grave{a}$-vis bending. ${ }^{15}$ Furthermore, the highly crosslinked planar oriented side presents an anisotropic susceptibility to bending due to the higher modulus along the nematic director versus that normal to the director. ${ }^{18}$ This presents a greater tendency to bending transverse to the nematic director in response to the biaxial stretching imposed on the homeotropic side. Hence, progressive development of strains with increasing temperatures leads to an increasing level of tensile strain on the homeotropic side and increasing compressive deformation transverse to the nematic director on the less-response planar oriented side. This response characterizes the behavior in the $0^{\circ}, 50^{\circ}$ and $90^{\circ}$ strips that were examined to manifest the observed accentuation of the native (as harvested) shapes of the samples. This underpins the magnification of the strains characterizing the native curvature.

The eversion of the strain state at the higher temperatures is counterintuitive to prior observations of the behavior of crosslinked LCP. ${ }^{6,8}$ In general, these systems are characterized by monotonic strain generation with increasing temperatures, where compressive strains are generated along the nematic director, while tensile strains are generated perpendicular to it. We eliminated residual polymerization as a potential mechanism underpinning the eversion and shape multiplicity observed here. Fourier transformed infrared (FTIR) spectroscopy in attenuated total reflectance mode was used to compare the conversion of the acrylate $\mathrm{C}=\mathrm{C}$ on the homeotropic side with respect to the planar side in the as-prepared films. Measuring the area under the $\mathrm{C}=\mathrm{C}$ peak $\left(\sim 1635 \mathrm{~cm}^{-1}\right)$, against the $\mathrm{C}=\mathrm{O}$ peak as the standard was used to calculate the conversion of the acrylate monomers. Fig. 6(a) illustrates the FTIR spectra of the monomers, the homeotropic side and the planar side. We find that the planar side that is not exposed to the atmosphere during curing is characterized by $\sim 99 \%$ conversion. On the other hand, the homeotropic side is characterized by $\sim 91 \%$ conversion, which can indicate some a gradation in the extent of polymerization through the thickness of the sample. Heat treatment of the samples at elevated temperatures can result in the progressive conversion of the unreacted acrylate moieties near the less polymerized homeotropic side. Concomitant polymerization shrinkage can produce compressive strains near the homeotropic side to drive the eversion that is observed in the samples. To track thermal polymerization, samples were heat treated under identical conditions to that in Fig. 4 and then subjected to FTIR analysis. Fig. 6(b) compares the spectra measured on the homeotropic side for the as-prepared samples at $25^{\circ} \mathrm{C} v s$. that heat treated to $200{ }^{\circ} \mathrm{C} . \mathrm{C}=\mathrm{C}$ peak was characterized, which shows that the conversion of the monomers increased marginally from $91 \%$ in the as-prepared sample to $\sim 93 \%$. This marginal increase in the degree of polymerization cannot explain the observed eversion.

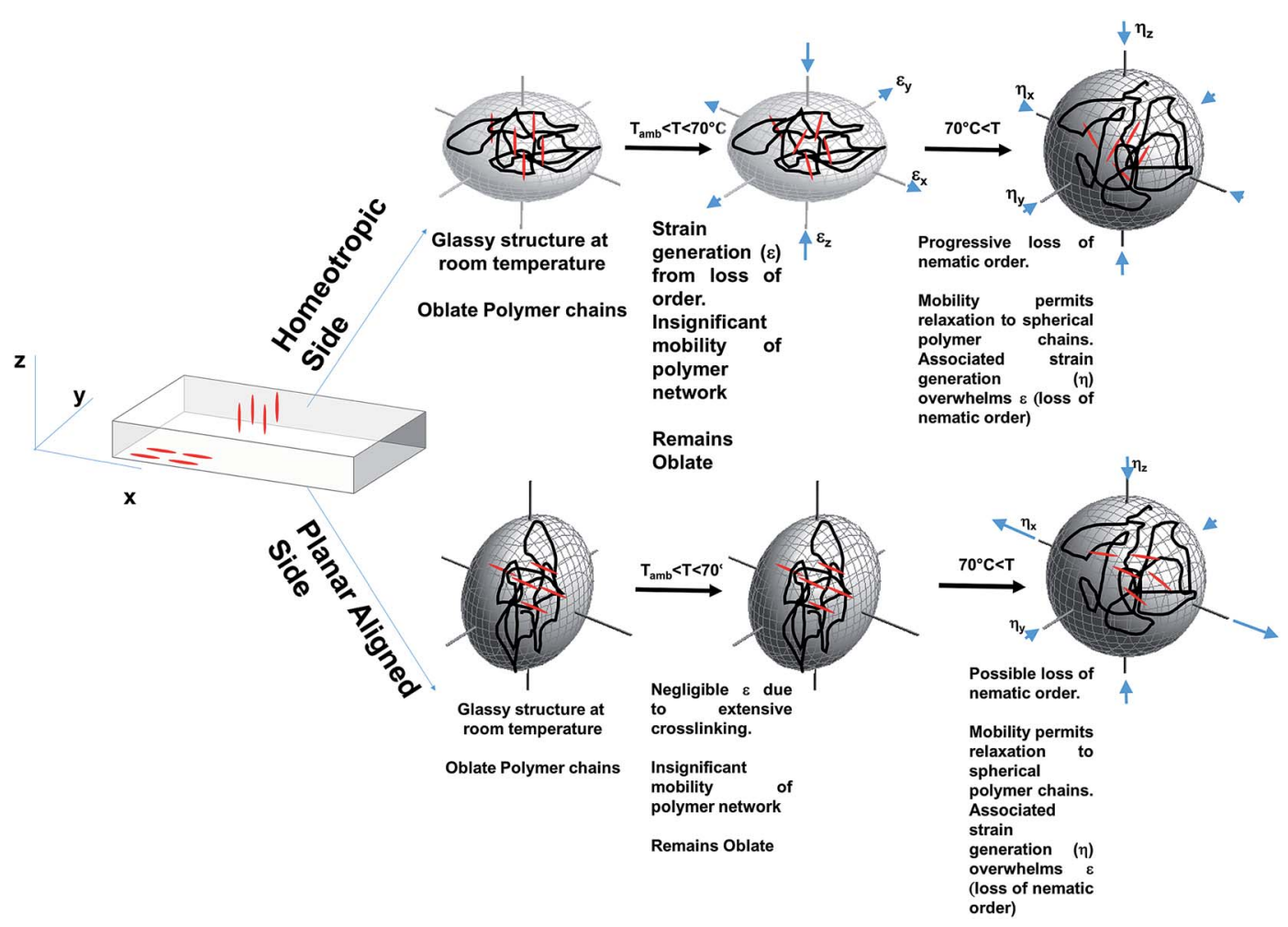

Fig. 5 Mechanisms underpinning the multimorphism in splayed liquid crystalline polymers in response to thermal treatment. 
- Homeotropic Side ${ }^{\Delta}$ Planar Side + Monomer (RMM34C)

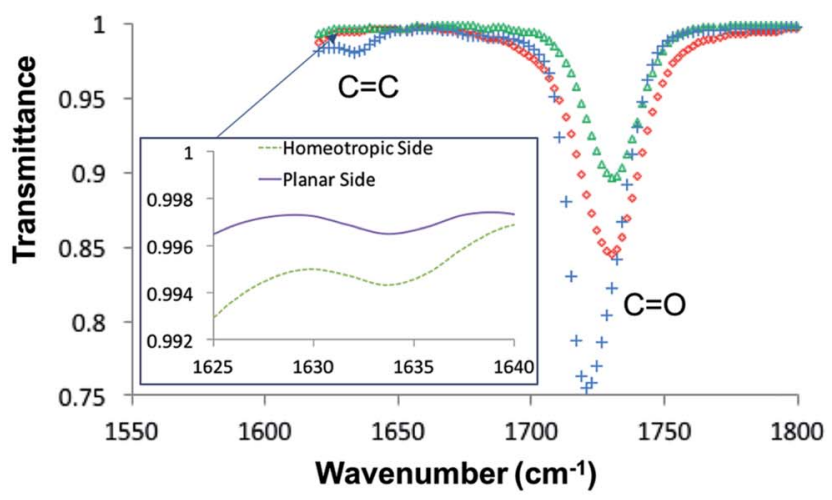

(a)

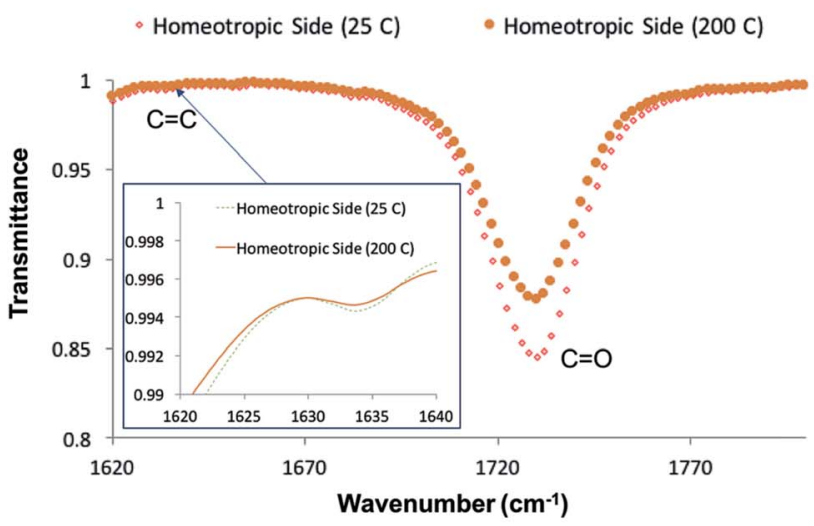

(b)

Fig. 6 ATR-FTIR spectra of the samples in the vicinity of the homeotropically and planar aligned surfaces. (a) As-prepared samples. (b) The spectrum acquired on the homeotropic side before and after heat treatment.

A prominent contribution to the observed eversion of the strain states at the elevated temperatures is the relaxation of the polymeric chains near the highly crosslinked planar oriented side. As noted earlier, when the sample is polymerized at an elevated temperature and then cooled to ambient conditions, the residual stresses emerge in the sample. The resulting elastic strains lead to the native shape when the sample is released from the substrate as illustrated in Fig. 1(b). In addition, on the highly crosslinked side that is polymerized with a planar alignment, a significant distortion of the polymer network can result as a result of the polymerization strains. Polymerization strains in acrylate-based mesogens are known to be the greatest in magnitude along the nematic director. ${ }^{19}$ Thus, a significant anisotropy can be inherited by the polymer network. The distortion of the polymer chains however, remains locked-in as a result of the cross linking and only exposure to high temperatures allows the release of these strains from relaxation of the polymer chains. Since, the polymerization strains produce oblate chains as illustrated in Fig. 5, their relaxation will eventually produce tensile strains along the nematic director on the planar side. This can explain why the samples are observed to develop significant tensile strains on the planar oriented state at elevated temperatures, with the principal tensile direction coinciding with the nematic director. While further research is needed to characterize this mechanism in detail, this can offer an explanation for the anomalous observations here. We note parenthetically that such crosslinked systems do not demonstrate any significant "soft elasticity" involving reorientation of the nematic director. ${ }^{6}$

\subsection{Actuation and morphing}

Eversion and multiplicity of the shapes as a function of the temperature offer opportunities for driving actuation and programming shape changes. For instance, the transformation of the shapes in Fig. 4(a) involves a switch-over in the principal axis of curvature, which can involve fast actuation if harnessed using suitable heating rates. In Fig. 7(a), a $0^{\circ}$ sample with dimensions of $1 \mathrm{~mm} \times 12 \mathrm{~mm} \times 15 \mu \mathrm{m}$ was excised and placed on a hot-plate set at $300{ }^{\circ} \mathrm{C}$. The sample initially remains static on the hot-plate, while being imaged with a $30 \mathrm{fps}$ camera. After a finite latency, the sample is found to jump spontaneously between 3 and $5 \mathrm{~mm}$ in height (see ESI Movie $\dagger$ ). The jump occurs within one frame of imaging, which indicates the ultrafast nature of the actuation at time-scales $\leq 30 \mathrm{~ms}$. The underlying premise is that by driving the shape transformations using sufficient heating can enable the utilization of the eversion of strain fields akin to snap-through mechanisms to enable high actuation rates. ${ }^{20}$ The switching of the principal curvatures can lead to the generation of power densities to achieve motility.

The observation of the tape spring geometry in the $0^{\circ}$ sample in Fig. 4(a), which can be modulated with temperature offers an opportunity for engineering active hinges. When a strip-shaped sample with a transverse curvature akin to the $0^{\circ}$ sample at ambient conditions is subjected to bending along its long axis, it typically localizes the curvature to form a fold. This is because, the slender geometry prefers to undergo localized bending instead of accommodating stretch. This is reminiscent of that observed with tape-springs that are bent. Fig. 7(b and c) illustrates the geometry adopted by a $0^{\circ}$ sample that is glued end-toend, length-wise in two distinct configurations. The dimensions of the strips are $35 \mathrm{~mm}$ (length) $\times 1 \mathrm{~mm}$ (width) and $15 \mu \mathrm{m}$ (thickness). If it were a flat strip, devoid of a native transverse curvature, a circular ring results. However, the transverse curvature forces localization of the bending strains, which is in turn a function of how the sample is deformed with respect to the native curvature. Such geometries have been examined for designing deployable aerospace structures and hinges. ${ }^{21}$ Adapting the nomenclature from this literature, Fig. 7(b) illustrates equal-sense bending, while Fig. $7(\mathrm{c})$ is opposite-sense bending. Here, the temperature dependent native curvature offers opportunities for tuning the structure.

Fig. 7(b) shows the creation of an oblong shape when the native curved sample is glued end-to-end to create a closed ring. The asymmetry results from the equal sense bending of 


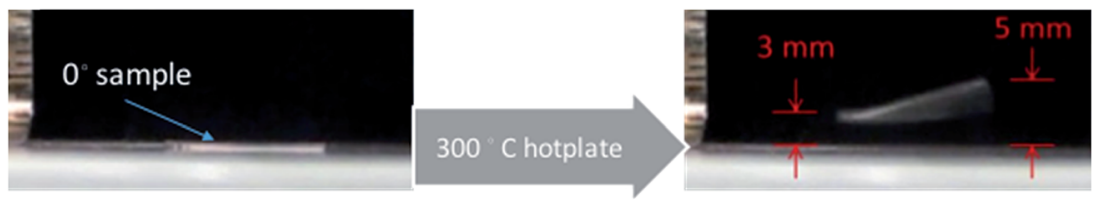

a)
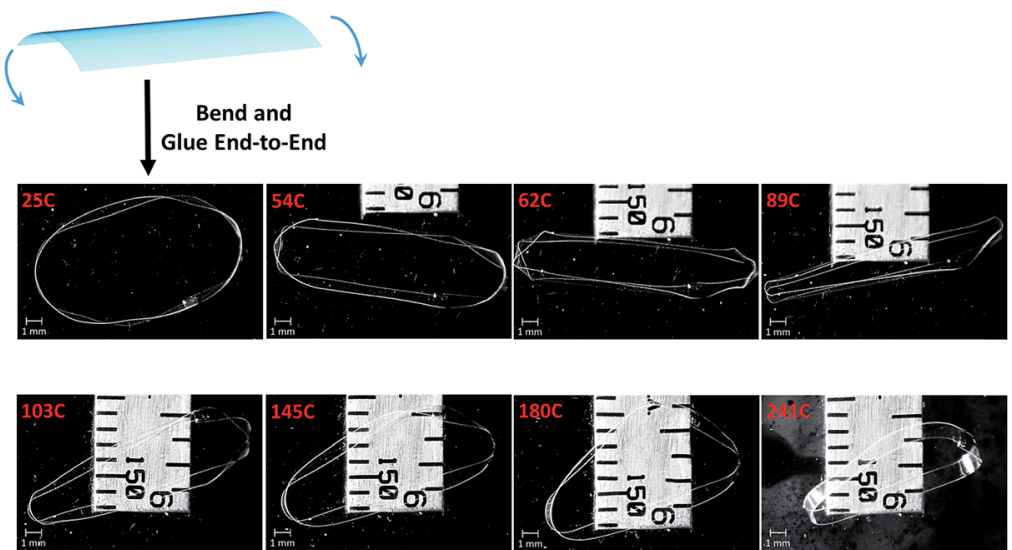

b)

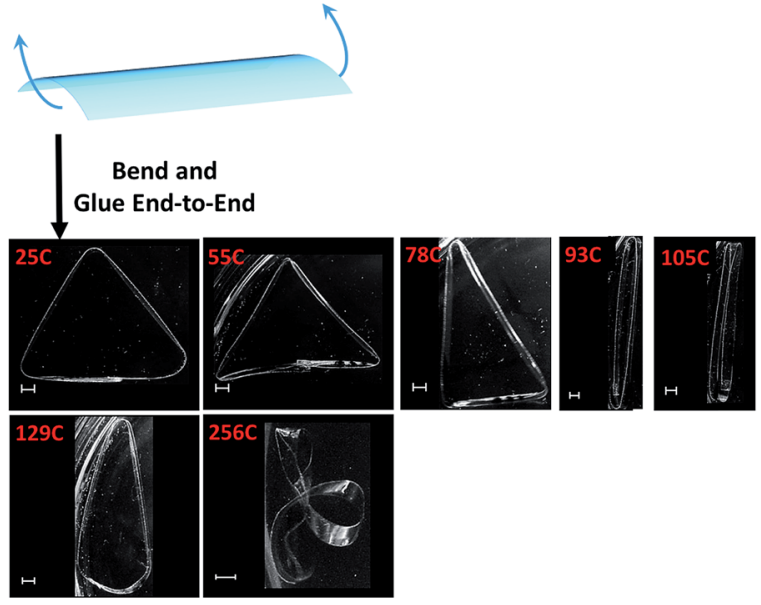

c)

Fig. 7 Actuation and morphing in native curved, splayed liquid crystalline polymers. (a) Spontaneous jumping of a $0^{\circ}$ sample placed on a hot plate (also see ESI Movie $\dagger$ ). (b) Geometric evolution of a ring fabricated by bending and gluing a $0^{\circ}$ sample in an equal sense configuration as a function of the temperature. The inset illustrates the gluing configuration. (c) Thermomechanical evolution of the geometry of a ring fabricated from a $0^{\circ}$ sample fabricated via opposite sense bending (also, see inset). The scale bars are $1 \mathrm{~mm}$.

a sample with a transverse curvature. When this ring is heated, the native curvature first increases akin to Fig. 4(a). This leads to the transformation of the geometry into a progressively lenticular shape, where the curvatures become increasingly localized. This trend continues until the relaxation of the transverse curvatures, which becomes prominent $>100{ }^{\circ} \mathrm{C}$. At this point, the lenticular shape regresses back into the oblong shape as illustrated in Fig. 7(b). The geometric evolution is much more dramatic in the Fig. 7(c) when rings that are fabricated in the opposite sense. The geometry at ambient conditions is characterized by significant localization of strains leading to the creation of a triangle-shaped structure. This structure remains 
stable under ambient conditions. When subjected to heating, a sharpening of the bends at the vertices is evident. This is because, as the transverse curvature becomes more pronounced, the slender geometry sequesters the bending to increasingly narrow zones to avoid incurring a stretching energy penalty due to Gaussian curvature. Greater the transverse curvature, the greater is the Gaussian curvature. The progressive heating leads to a spontaneous transformation of the equilateral triangle into an isosceles triangle at $78{ }^{\circ} \mathrm{C}$. Progressive heating leads to the relaxation of the transverse curvatures, which converts the triangle into a lenticular shape. This is because of reduction in the Gaussian curvature that results from the reduced transverse curvature. However, as the temperature further increases, we see the development of a 3dimensional supercoiled structure at $256{ }^{\circ} \mathrm{C}$. At high temperatures, we find that the $0^{\circ}$ samples undergo eversion to generate a curvature orthogonal to that in the native ambient state. In these samples, the fixturing of the samples end-to-end remains unchanged, even while the sample attempts to adopt an everted geometry. Under these conditions supercoiling spontaneously emerges to create a trifoil shape that is illustrated in Fig. 7(c) at $256{ }^{\circ} \mathrm{C}$. The evolution of this geometry is likely related to the mechanics identified in ref. 22 and 23, where the development of the curvatures under topological confinement manifests the dramatic shape diversity.

\section{Conclusion}

Polymerizing liquid crystalline monomers on rubbed substrates, while being exposed to air offers a means for endowing a gradation of both the nematic directors and the cross link densities. This interplay endows a functional gradation to these films and enables the realization of a multiplicity in shape selection as a function of heat treatment. The asprepared samples are characterized by a native curvature at ambient conditions with the planar oriented sides characterized by a compressive strain and the homeotropic side illustrating a tensile state. When strips are excised from them, they are found to adopt a range of geometries as a function of the offset angle with respect to the planar oriented side. Thermal treatment of these strips under quasistatic conditions first results in a magnification of the strain state characterizing the native curvatures. However, progressive increase in the temperatures results in an eversion of the samples, where they adopt curvature along an orthogonal axis. The interplay of the hierarchical variation of the nematic director through the thickness of the sample and the gradation in the crosslink density is related to the multiplicity of shapes. Prototypical exploitation of these samples as actuators and as morphing structures is explored in simple configurations. A suitably chosen strip when placed on a preheated hot-plate is found to spontaneously jump off the surface as a result of the eversion. When the samples are topologically confined into rings by gluing them end-to-end, localization of strains results enabling a folded structure. Subsequent heat treatment leads to the evolution of the folds as a function of the temperature. This multiplicity can offer an array of opportunities for engineering morphing devices and actuators by exploiting the functional gradation in liquid crystalline polymers.

\section{Acknowledgements}

We gratefully acknowledge support from the Air Force Office of Scientific Research and the National Science Foundation (\#1435489).

\section{References}

1 A. Lendlein and S. Kelch, Angew. Chem., Int. Ed., 2002, 41, 2034-2057.

2 C. Liu, H. Qin and P. Mather, J. Mater. Chem., 2007, 17, 15431558.

3 P. T. Mather, X. Luo and I. A. Rousseau, Annu. Rev. Mater. Res., 2009, 39, 445-471.

4 T. H. Ware, M. E. McConney, J. J. Wie, V. P. Tondiglia and T. J. White, Science, 2015, 347, 982-984.

5 M. E. McConney, A. Martinez, V. P. Tondiglia, K. M. Lee, D. Langley, I. I. Smalyukh and T. J. White, Adv. Mater., 2013, 25, 5880-5885.

6 K. M. Lee, T. J. Bunning and T. J. White, Adv. Mater., 2012, 24, 2839-2843.

7 Y. Sawa, F. Ye, K. Urayama, T. Takigawa, V. Gimenez-Pinto, R. L. Selinger and J. V. Selinger, Proc. Natl. Acad. Sci. U. S. A., 2011, 108, 6364-6368.

8 G. N. Mol, K. D. Harris, C. W. Bastiaansen and D. J. Broer, Adv. Funct. Mater., 2005, 15, 1155-1159.

9 M. L. Smith, K. M. Lee, T. J. White and R. A. Vaia, Soft matter, 2014, 10, 1400-1410.

10 J. J. Wie, K. M. Lee, T. H. Ware and T. J. White, Macromolecules, 2015, 48, 1087-1092.

11 J. J. Wie, K. M. Lee, M. L. Smith, R. A. Vaia and T. J. White, Soft Matter, 2013, 9, 9303-9310.

12 C. L. van Oosten, C. W. Bastiaansen and D. J. Broer, Nat. Mater., 2009, 8, 677-682.

13 S. Iamsaard, S. J. Aßhoff, B. Matt, T. Kudernac, J. J. Cornelissen, S. P. Fletcher and N. Katsonis, Nat. Chem., 2014, 6, 229-235.

14 M. Guvendiren, S. Yang and J. A. Burdick, Adv. Funct. Mater., 2009, 19, 3038-3045.

15 S. Armon, E. Efrati, R. Kupferman and E. Sharon, Science, 2011, 333, 1726-1730.

16 Y. Xia, F. Serra, R. D. Kamien, K. J. Stebe and S. Yang, Proc. Natl. Acad. Sci. U. S. A., 2015, 112, 15291-15296.

17 W.-Y. Wu, C.-C. Wang and A. Y. Fuh, Opt. Express, 2008, 16, 17131-17137.

18 C. Van Oosten, K. Harris, C. Bastiaansen and D. Broer, Eur. Phys. J. E: Soft Matter Biol. Phys., 2007, 23, 329-336.

19 R. Hikmet, B. Zwerver and D. Broer, Polymer, 1992, 33, 89-95.

20 H. Lee, C. Xia and N. X. Fang, Soft Matter, 2010, 6, 4342-4345.

21 K. Seffen and S. Pellegrino, Proc. R. Soc. London, Ser. A, 1999, 455, 1003-1048.

22 B. D. Coleman and D. Swigon, Journal of Elasticity, 2000, 60, 173-221.

23 P.-O. Mouthuy, M. Coulombier, T. Pardoen, J.-P. Raskin and A. M. Jonas, Nat. Commun., 2012, 3, 1290. 